\title{
Properties of $\alpha$-Brass Nanoparticles II: Structure and Composition
}

\author{
Jan Weinreich, ${ }^{\dagger}$ Martín Leandro Paleico, ${ }^{\dagger}$ and Jörg Behler, ${ }^{*}, \ddagger$ \\ †Universität Göttingen, Institut für Physikalische Chemie, Theoretische Chemie, \\ Tammannstraße 6, 37077 Göttingen, Germany \\ $\ddagger$ International Center for Advanced Studies of Energy Conversion (ICASEC), Universität \\ Göttingen, Tammannstraße 6, 3707 ry Göttingen, Germany \\ E-mail: joerg.behler@uni-goettingen.de
}

\section{Supplemental Information}

\subsection{Crystalline Surfaces}

\subsubsection{Position of Zinc in Copper Slabs}

In order to understand the energetically most favorable positions of $\mathrm{Zn}$ atoms at the surfaces in large brass NPs, we have investigated the stability of monolayers of $\mathrm{Zn}$ atoms in 13-layer $1 \times 1$ $\mathrm{Cu}(111), \mathrm{Cu}(100)$ and $\mathrm{Cu}(110)$ slabs. Fig. S1 shows the relative energies obtained with DFT and the HDNNP as a function of the position of the $\mathrm{Zn}$ layer in the slab. The slabs have been fully relaxed in these calculations. We find that for all three surfaces the lowest-energy configuration corresponds to a position of the Zn layer at the surface, which is a consequence of maximizing the number of bonds between the copper atoms, which are stronger than bonds involving Zn atoms. The energy differences between the zinc layer in the center of the slab and at the surface are given in Table S1.

\subsubsection{Surface Patterns of Brass Slabs}

In order to investigate the surface structure of periodic brass slabs as a reference for the brass NPs, we have performed MMC-SGCE simulations with a fixed lattice where each supercell contains 512 atoms.

The MMC-SGCE simulation temperature is
Table S1: Energy differences between two slab configurations with a $\mathrm{Zn}$ layer at the surface and in the center, respectively, for three surfaces (s. Fig. S1).

\begin{tabular}{ccc} 
Surface & $\begin{array}{c}\text { HDNNP } \\
\text { (meV/atom) })\end{array}$ & $\begin{array}{c}\text { DFT } \\
\text { (meV/atom) }\end{array}$ \\
\hline$(111)$ & 20.2 & 19.6 \\
$(100)$ & 12.8 & 14.2 \\
$(110)$ & 20.4 & 21.6 \\
\hline
\end{tabular}

kept constant at $T=200 \mathrm{~K}$ while the chemical potential differences $\Delta \mu$ are altered.

In Fig. S2 we show the obtained average Zn atom fractions $\left\langle x_{\mathrm{Zn}}\right\rangle$ for the (111), (110) and (100) surfaces as well as for the bulk. For all systems, the zinc contents increases with more negative $\Delta \mu$ but the slope strongly depends on the chemical potential difference, and for example at about $\Delta \mu=-2.4 \mathrm{eV}$ the composition changes rapidly, while at about $\Delta \mu=-2.2 \mathrm{eV}$ there is only a small change of $\left\langle x_{\mathrm{Zn}}\right\rangle$. This behavior resembles the composition plateaus observed for brass NPs (Fig. 4 in main text). Interestingly, for $\Delta \mu=-2.0 \mathrm{eV}$ the incorporation of $\mathrm{Zn}$ shows an earlier onset than for the (111) and (100) surfaces, which might be related to the stability of one-dimensional $\mathrm{Zn}$ atom rows (Fig. S3b) at the surface in analogy to the Zn chains found in NPs (Fig. 9 in main text).

The composition of the surfaces is shown in Fig. S3 for a chemical potential difference of 


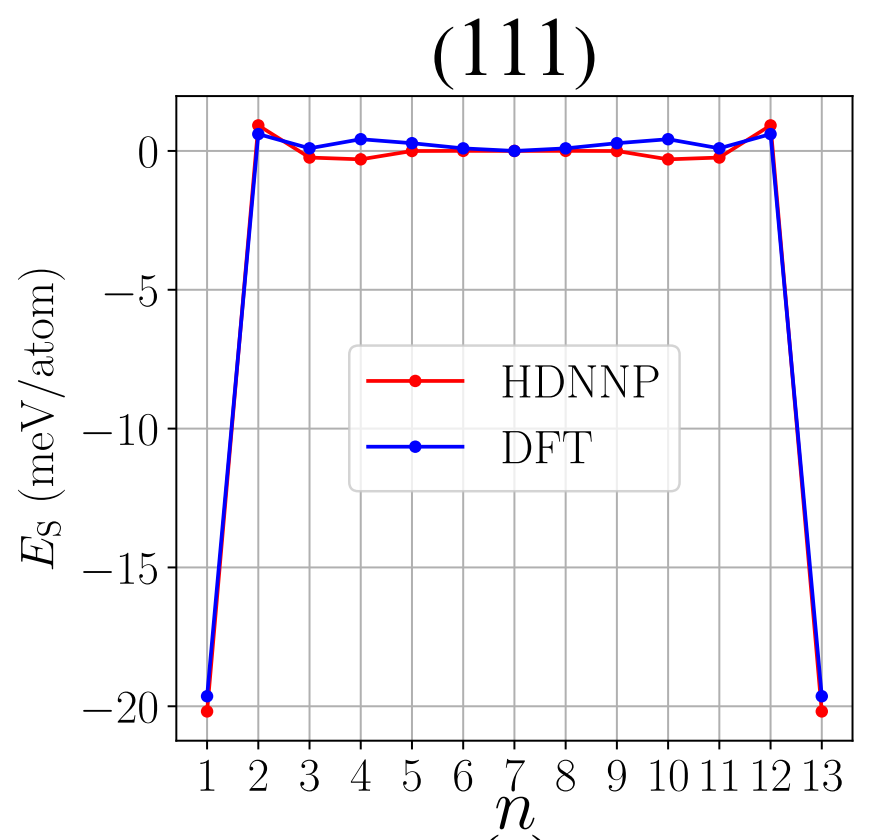

(a)

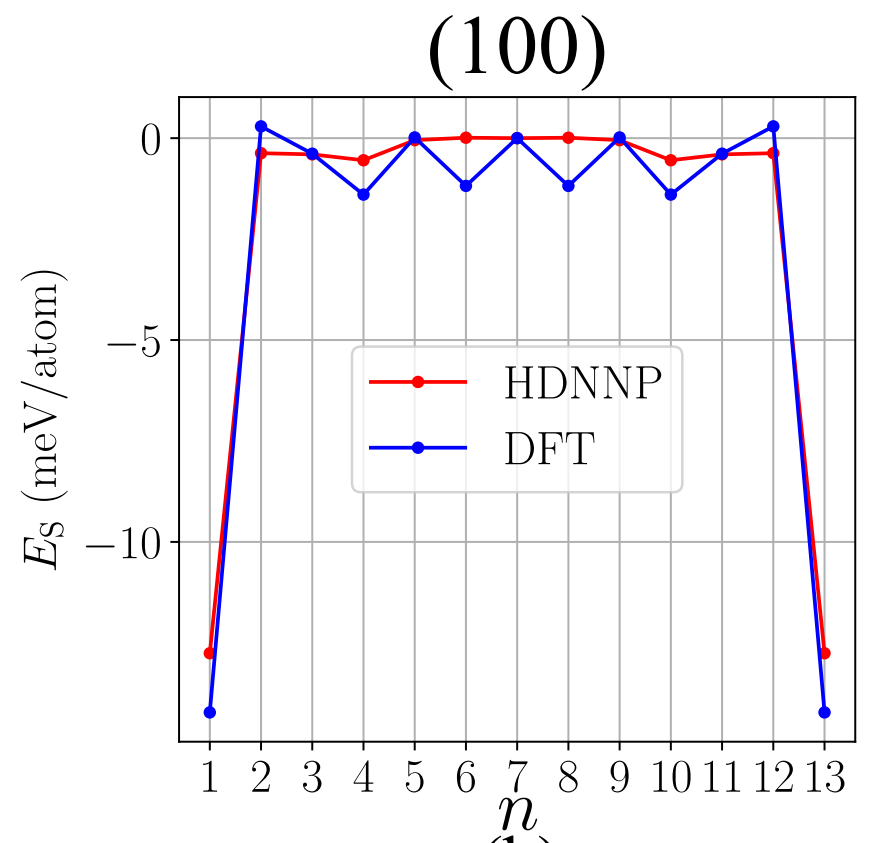

(b)

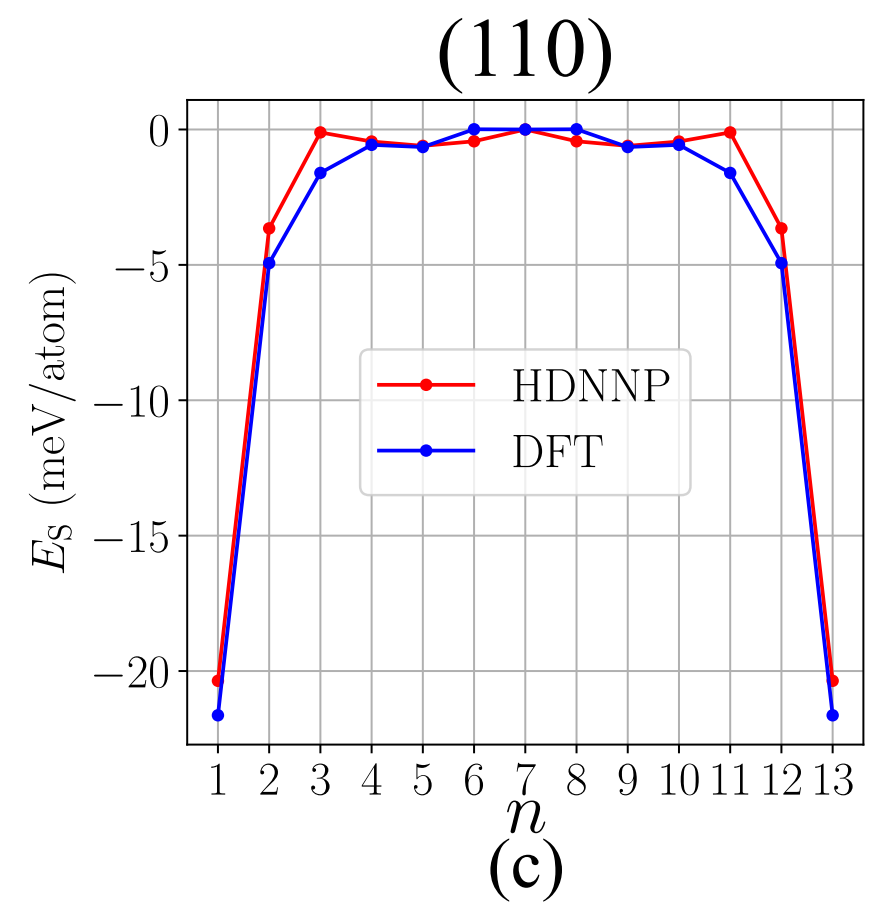

Figure S1: Panels (a), (b) and (c) show the relative energies per atom of 13 -layer $1 \times 1 \mathrm{Cu}(111)$, $\mathrm{Cu}(100)$ and $\mathrm{Cu}(110)$ slabs containing a single layer of Zn atoms as a function of the layer number $n$ indicating the positions of the $\mathrm{Zn}$ monolayer. The energies have been determined for relaxed slabs using DFT (red) and the HDNNP (blue). The first and the last points of each graph $(n=1$ and $n=13$ ) correspond to configurations in which $\mathrm{Zn}$ is at the surface. As only energy differences are relevant, the DFT and HDNNP energies have been aligned for the structure containing Zn in the central layer of the respective slab.

$\Delta \mu=-2.37 \mathrm{eV}$ at the end of the stability plateau just before the rapid increase in Zn contents. Overall, the observed patterns are similar to those found on the facets of the brass NPs. 


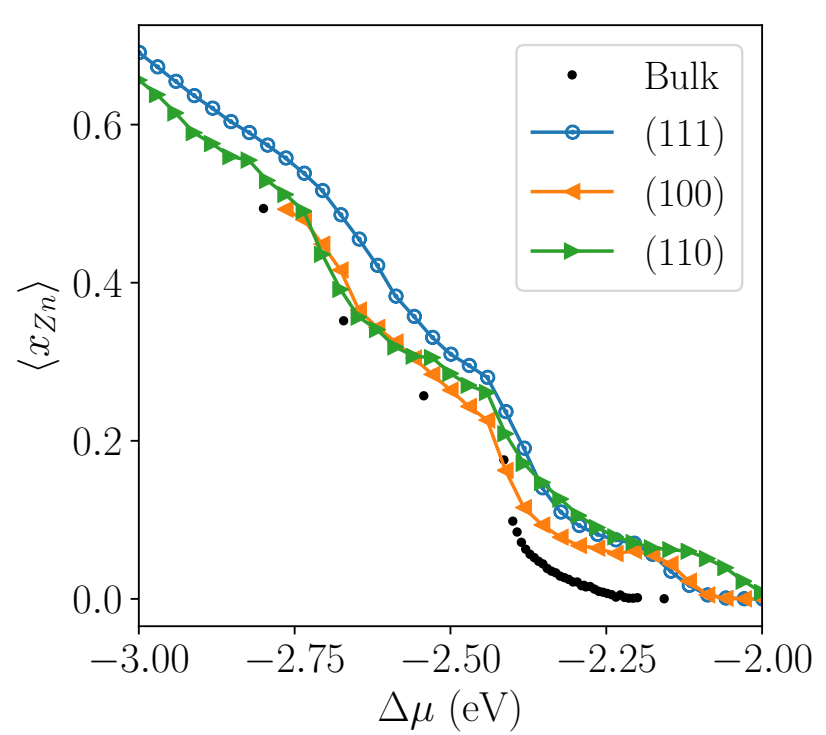

Figure S2: Average Zn atom fraction $\left\langle x_{\mathrm{Zn}}\right\rangle$ at $T=200 \mathrm{~K}$ in the SGCE at different chemical potential differences $\Delta \mu$ for the (100), (110) and (111) surfaces of brass and the bulk.

\subsection{Brass Nanoparticles}

\subsubsection{Temperature Dependence of Composition}

The influence of temperature $T$ on the $\mathrm{Zn}$ atom fractions $\left\langle x_{\mathrm{Zn}}\right\rangle$ and on the distribution of $\mathrm{Cu}$ and $\mathrm{Zn}$ atoms in the MMC-SGCE simulations is shown in Fig. S4 for two different chemical potential differences $\Delta \mu$. As before the lattice is fixed in these simulations. As expected, for increasing temperatures the potential energy difference of different element distributions becomes less dominant resulting in less pronounced element-specific occupation patterns (Figs. S4b and d). For the same reason, at a rather low chemical potential of $\mathrm{Zn}$ (less negative value of $\Delta \mu$ ) in Fig. S4a the $\mathrm{Zn}$ atom fraction increases with $T$, while it decreases at a higher Zn chemical potential (Fig. S4c). At $T=150 \mathrm{~K}$ and $\Delta \mu=-2.4 \mathrm{eV}$ we find a remarkable exception at the composition $x_{\mathrm{Zn}}=25 \%$ Zn for the energetically very favorable $L_{12}$ bulk phase $^{1,2}$ (Fig. S4a). For higher temperatures $T$ we find that the $\mathrm{Zn}$ concentration will drop again, likely because the symmetric arrangement of atoms in the $L_{12}$ cannot be stable at higher temperatures. Interestingly for increas- ingly higher temperatures the Zn concentration will increase again, because a higher $\mathrm{Zn}$ fraction corresponds to a higher cohesive energy ${ }^{1}$ which corresponds to higher temperatures in the MMC-SGCE simulations.

\subsubsection{Constant Temperature and Relax- ation}

In case of simulations with fixed lattices, in particular the energies of structures with high $\mathrm{Zn}$ contents will be overestimated. To investigate the role of lattice relaxation, which typically yields the closest local minimum but no substantial global structural rearrangements, we have performed MMC SGCE simulations including a full geometry optimization in each step. As this optimization decreases the energy in particular if rather large Zn atoms are incorporated in the system, we find that $\left\langle x_{\mathrm{Zn}}\right\rangle$ for a given $\Delta \mu$ is higher than for a fixed lattice (Fig. S5a). Thus a smaller $\Delta \mu$ value is required to reach a given $\left\langle x_{\mathrm{Zn}}\right\rangle$, which is preferentially located in the topmost atomic layer of the NPs. Moreover, the average next nearest neighbour distances $d_{\mathrm{nn}}$ between all atoms predicted by the HDNNP increases with $x_{\mathrm{Zn}}$ as shown in Fig. S5b. In comparison to the bulk phase the expansion of the NPs as a function of $x_{\mathrm{Zn}}$ is shifted towards smaller $d_{\mathrm{nn}}$, because of the smaller interatomic distances at the surface caused by inwards relaxation of the undercoordinated topmost layer leading to a larger $d_{\mathrm{nn}}$ for smaller NPs with a high surface-to-bulk ratio. For the 459 and 1103 atom NPs $d_{\text {nn }}$ initially increases much slower than for bulk $\alpha$ brass since Zn accumulates at the surface. This extension of this surface incorporation depends on the surface-volume ratio and thus on the NP size. For instance, a $\mathrm{Zn}$ atom fraction of at least $14 \%$ is necessary for the 1103 atom NP with $38 \%$ surface atoms and about $16 \%$ for the 459 atom NP with $51 \%$ surface atoms to achieve a bulk like linear increase of $d_{\mathrm{nn}}$ with expansion coefficients $b^{\text {ex. }}$ (Fig. S5b). As can be seen in the linear regressions, the respective next neighbour expansion coefficients are similar to the value for bulk brass, although at the mentioned concentrations the whole surface is 


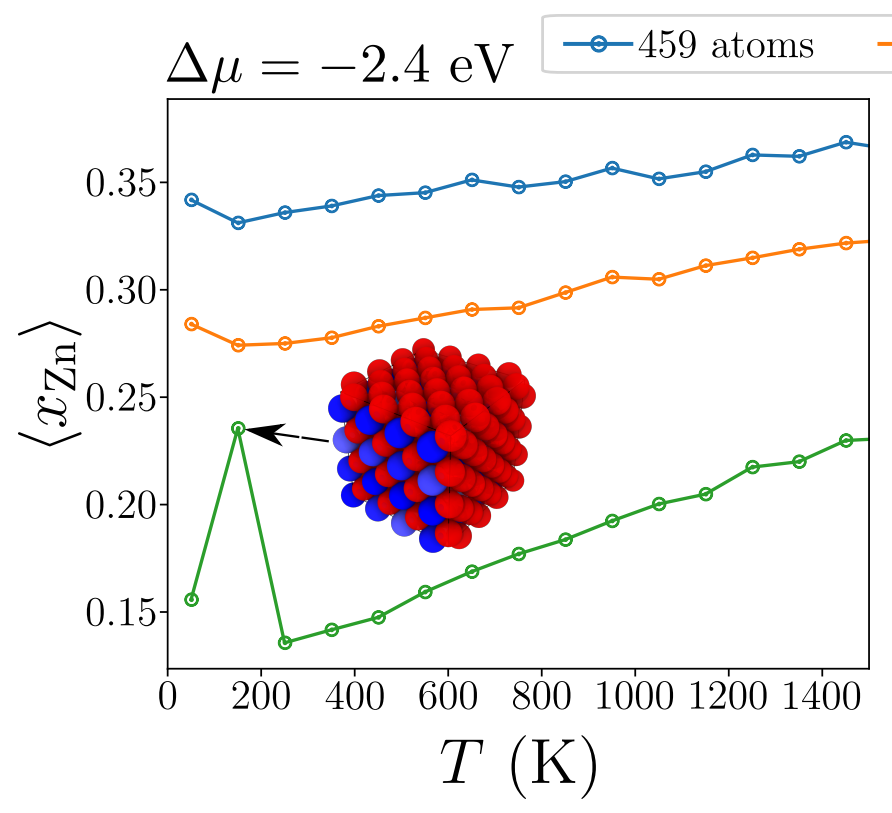

(a)

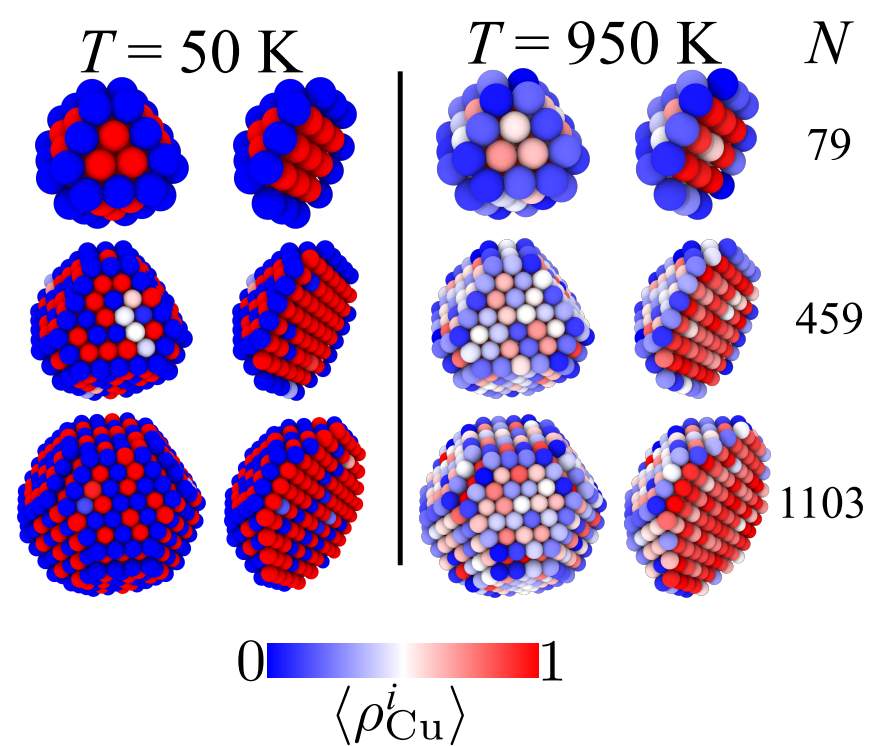

(b)

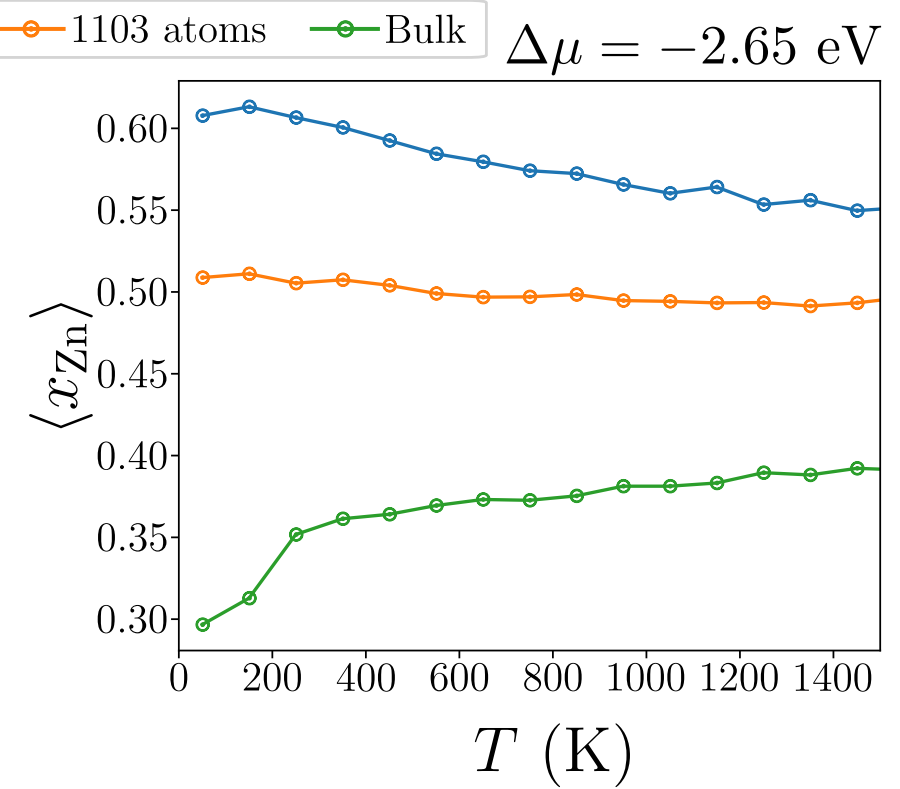

(c)

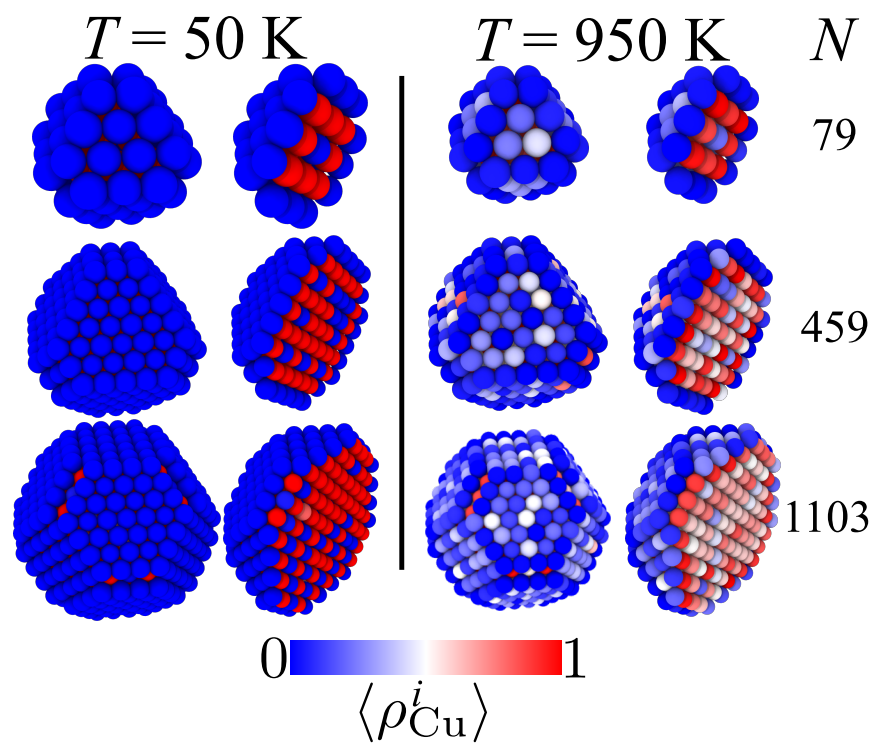

(d)

Figure S3: Brass (100), (110) and (111) surfaces resulting from MMC-SGCE simulations using the HDNNP at $T=200 \mathrm{~K}$ and $\Delta \mu=-2.37 \mathrm{eV}$ with a fixed lattice. The lateral supercells used in the simulations are highlighted by black rectangles. Copper atoms are shown in brown, zinc atoms in grey.

not yet occupied by Zn. The reason for this observation is that $\mathrm{Zn}$ incorporation in the interior of the NPs sets before the surface is completely saturated with Zn.

\subsubsection{Melting of Brass Nanoparticles}

We have performed an additional test of the melting point relation $T_{m}\left(N, x_{\mathrm{Zn}}\right)$ as a func-
Table S2: Composition dependent melting constant $k_{c}$ obtained from simulations of brass nanoparticles with different number of atoms $N$ and the bulk.

\begin{tabular}{cccc} 
& $N=165$ & $N=1103$ & Bulk \\
\hline$k_{c}(\mathrm{~K})$ & $5.2 \pm 0.62$ & $5.37 \pm 0.2$ & $5.34 \pm 0.1^{3}$ \\
\hline
\end{tabular}




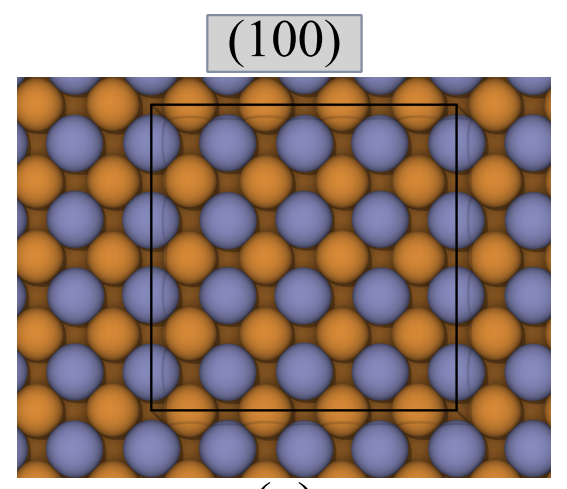

(a)

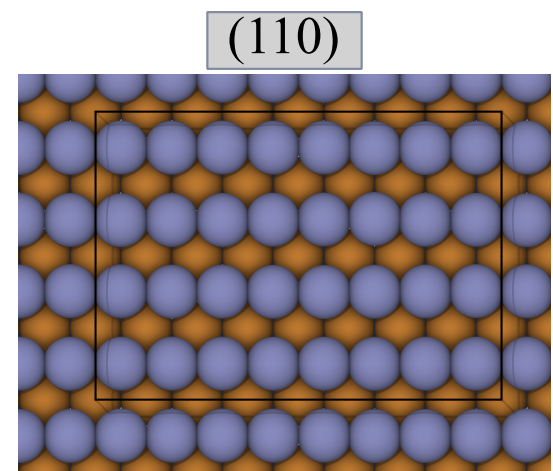

(b)

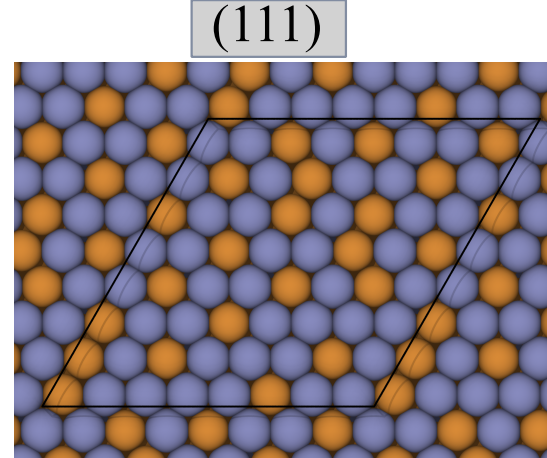

(c)

Figure S4: Composition and element distributions of brass nanoparticles for different temperatures and chemical potential differences with a fixed lattice. Panels (a) and (c) show the Zn atom fractions $\left\langle x_{\mathrm{Zn}}\right\rangle$ as a function ot $T$ for NPs containing 459 and 1103 atoms as well as for bulk brass for two different chemical potential differences $\Delta \mu=-2.4 \mathrm{eV}$ and $-2.65 \mathrm{eV}$. Panels (b) and (d) display the surface views and cross sections of the site occupations $\left\langle\rho_{\mathrm{Cu}}^{i}\right\rangle$ by copper for three NP sizes for the same respective chemical potential differences at $T=50 \mathrm{~K}$ and $T=950 \mathrm{~K}$.

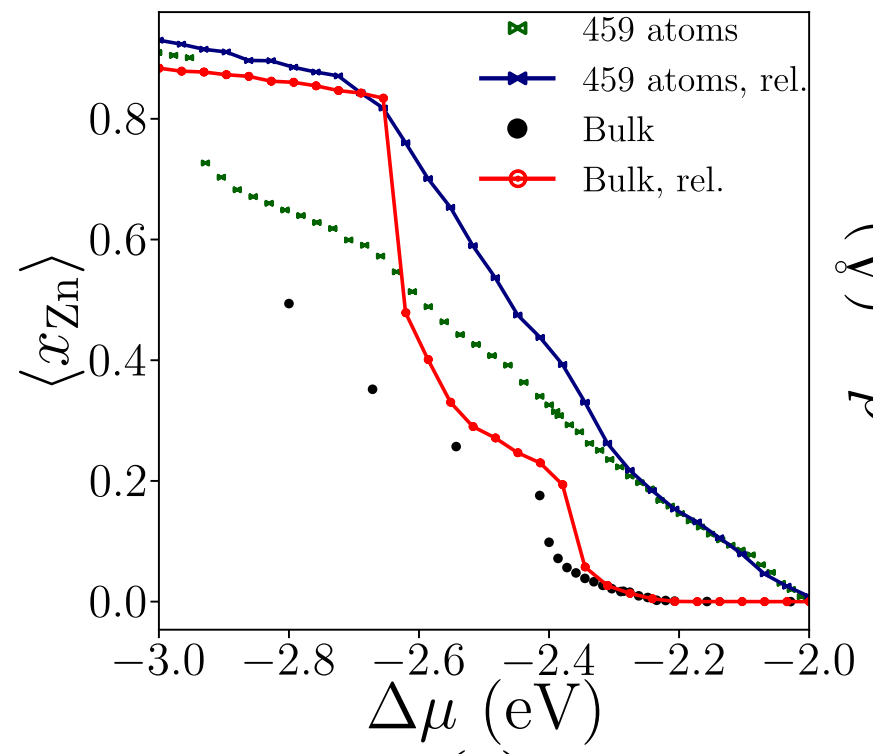

(a)

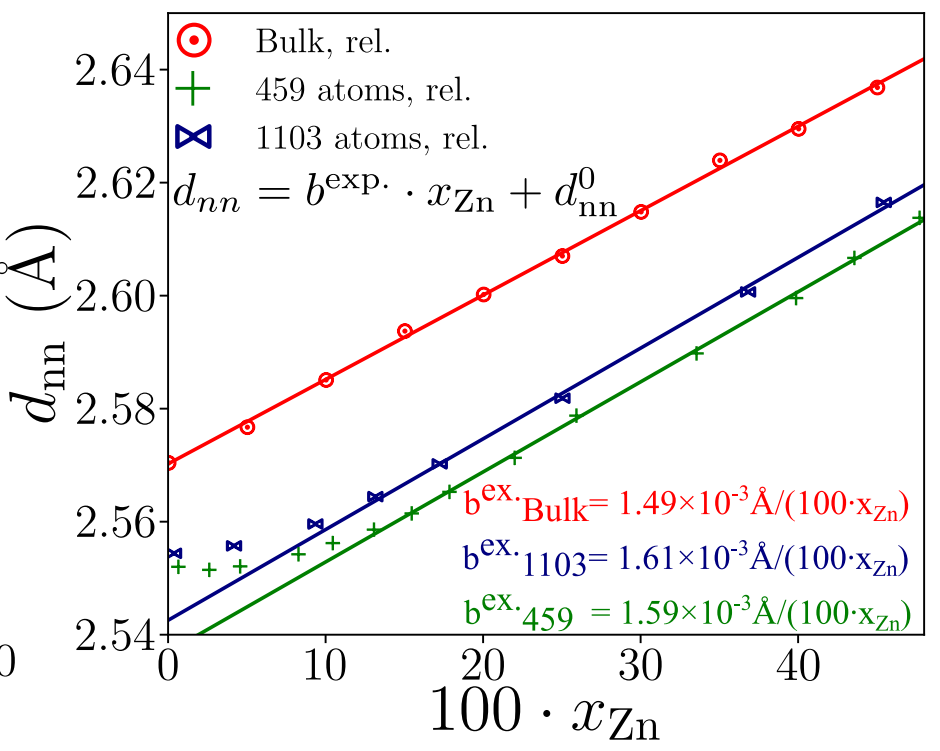

(b)

Figure S5: Average Zn atom fractions $\left\langle x_{\mathrm{Zn}}\right\rangle$ for brass clusters of different sizes as a function of the chemical potential difference $\Delta \mu$ at $T=200 \mathrm{~K}$ (a). As shown for the NPs containing 459 atoms, for a given $\Delta \mu$ the relaxation of the atomic positions results in higher Zn contents since lattice strain is reduced. Average next neighbor distances $d_{\mathrm{nn}}$ between all pairs of atoms in the NPs and relaxed bulk for fixed Zn atom fractions $x_{\mathrm{Zn}}$ in (b). Linear regression can be used to determine the

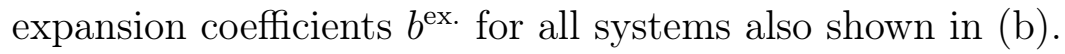

tion of the $\mathrm{Zn}$ atom fraction $x_{\mathrm{Zn}}$ (s. Eq. 6 in the MS) for a Wulff-shaped brass nanoparticle of $N=165$ atoms with up to $40 \% \mathrm{Zn}$ content to understand if and how the size of the nanoparticle affects the composition dependent melting constant $k_{c}$ (s. Eq. 6 in the MS). Us- ing the Lindemann melting criterion ${ }^{1}$ we find that $T_{m}$ decreases linearly with $x_{\mathrm{Zn}}$ as expected (s. Fig. S6). However, we find three apparent outliers for $x_{\mathrm{Zn}}=30,35,40 \%$. After removing these points from the linear regression we find a good agreement of $k_{c}$ with the value re- 


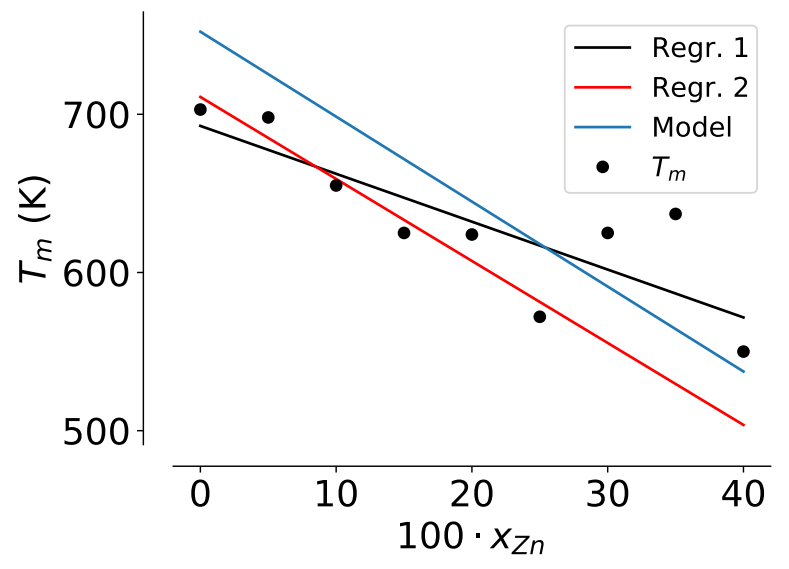

Figure S6: Melting points $T_{m}$ of a Wulff shaped $N=165$ atom nanoparticle as a function of the zinc atom fraction $x_{\mathrm{Zn}}$ as well as linear regression (Regr. 1) to the data and the predicted melting points using Eq. (6) (Model) from the manuscript. The linear regression model Regr. 2 results from neglecting the last three datapoints which are apparent outliers.

ported in the MS i.e. the composition dependent melting constant is primarily determined by the melting of the corresponding bulk phase and the bulk cohesive energy (s. table S2 for a full comparison). 


\section{References}

(1) Weinreich, J.; Römer, A.; Paleico, M. L.; Behler, J. Properties of $\alpha$-Brass Nanoparticles. 1. Neural Network Potential Energy Surface. J. Phys. Chem. C 2020, 124, 12682-12695.

(2) Müller, S.; Zunger, A. Structure of Ordered and Disordered $\alpha$-Brass. Phys. Rev. B 2001, 63, 094204.

(3) Lide, D. R. CRC Handbook of Chem. and Phys., 85th ed.; CRC Press, Boca Raton FL, 2005. 